\title{
Défis et principales orientations d'un projet de recherche ancré dans des problématiques socio-environnementales locales et destiné à des adultes peu alphabétisés en situation d'exclusion Challenges and the principal orientations of a research project on local socio-environmental problems conducted with adult literacy students in situations of exclusion \\ Retos y orientaciones principales de un proyecto de investigación arraigado en problemáticas socio-ambientales locales y destinado a adultos poco alfabetizados en situación de exclusión
}

\section{Carine Villemagne}

Volume 37, numéro 2, automne 2009

Vivre ensemble, sur Terre

URI : https://id.erudit.org/iderudit/038819ar

DOI : https://doi.org/10.7202/038819ar

Aller au sommaire du numéro

\section{Éditeur(s)}

Association canadienne d'éducation de langue française

ISSN

0849-1089 (imprimé)

1916-8659 (numérique)

Découvrir la revue

Citer cet article

Villemagne, C. (2009). Défis et principales orientations d'un projet de recherche ancré dans des problématiques socio-environnementales locales et destiné à des adultes peu alphabétisés en situation d'exclusion. Éducation et francophonie, 37(2), 119-131. https://doi.org/10.7202/038819ar

\section{Résumé de l'article}

Vivre ensemble dans nos sociétés occidentales ne veut pas nécessairement dire être en relation avec d'autres. Un quotidien très solitaire peut être vécu par des individus en plein coeur de villes densément peuplées. Certaines populations sont susceptibles plus que d'autres de vivre isolées, sans espace d'expression ni de liens de solidarité, dans des milieux de vie de piètre qualité environnementale. Or, ce sont ces populations défavorisées qui sont les plus confrontées aux désordres sociaux et écologiques, considérant leur situation comme un «mal» acceptable.

Dans le cadre d'un projet de recherche mené par trois partenaires (deux organismes communautaires et une université), l’objectif général poursuivi consiste en la conception et en l'expérimentation d'un programme éducatif, " Alphabétisation et éducation relative à l'environnement des adultes ». Un tel programme vise l'engagement social et environnemental, dans leur milieu de vie, de personnes adultes peu alphabétisées. La démarche de recherche choisie est celle d'une " recherche de développement d'objet éducatif » où les données recueillies tout au long de la recherche renseignent autant sur son processus que sur ses résultats.

La recherche étant à ses débuts, les trois partenaires travaillent à concevoir le programme éducatif en se basant sur leurs expériences pratiques et sur les écrits théoriques recensés et analysés. À cette étape de la recherche, il apparaît que plusieurs défis sont à relever pour mettre en oeuvre le programme " Alphabétisation et éducation relative à l'environnement des adultes ». Nous en examinerons quelques-uns, tantôt de nature théorique, tantôt de nature pratique. Nous présenterons ensuite les grandes orientations que les organismes collaborateurs souhaitent donner à ce programme, orientations qui ont émergé d'un premier groupe de discussion.

Tous droits réservés $@$ Association canadienne d’éducation de langue française, Ce document est protégé par la loi sur le droit d’auteur. L’utilisation des 2009 services d'Érudit (y compris la reproduction) est assujettie à sa politique d'utilisation que vous pouvez consulter en ligne.

https://apropos.erudit.org/fr/usagers/politique-dutilisation/ 


\section{Défis et principales orientations d'un projet de recherche ancré dans des problématiques socio- environnementales locales et destiné à des adultes peu alphabétisés en situation d'exclusion}

\section{Carine VILLEMAGNE}

Université de Sherbrooke, Québec, Canada

\section{RÉSUMÉ}

Vivre ensemble dans nos sociétés occidentales ne veut pas nécessairement dire être en relation avec d'autres. Un quotidien très solitaire peut être vécu par des individus en plein cœur de villes densément peuplées. Certaines populations sont susceptibles plus que d'autres de vivre isolées, sans espace d'expression ni de liens de solidarité, dans des milieux de vie de piètre qualité environnementale. Or, ce sont ces populations défavorisées qui sont les plus confrontées aux désordres sociaux et écologiques, considérant leur situation comme un «mal» acceptable.

Dans le cadre d'un projet de recherche mené par trois partenaires (deux organismes communautaires et une université), l'objectif général poursuivi consiste en la 
conception et en l'expérimentation d'un programme éducatif, "Alphabétisation et éducation relative à l'environnement des adultes». Un tel programme vise l'engagement social et environnemental, dans leur milieu de vie, de personnes adultes peu alphabétisées. La démarche de recherche choisie est celle d'une «recherche de développement d'objet éducatif» où les données recueillies tout au long de la recherche renseignent autant sur son processus que sur ses résultats.

La recherche étant à ses débuts, les trois partenaires travaillent à concevoir le programme éducatif en se basant sur leurs expériences pratiques et sur les écrits théoriques recensés et analysés. À cette étape de la recherche, il apparaît que plusieurs défis sont à relever pour mettre en œuvre le programme «Alphabétisation et éducation relative à l'environnement des adultes ». Nous en examinerons quelquesuns, tantôt de nature théorique, tantôt de nature pratique. Nous présenterons ensuite les grandes orientations que les organismes collaborateurs souhaitent donner à ce programme, orientations qui ont émergé d'un premier groupe de discussion.

\section{ABSTRACT}

\section{Challenges and the principal orientations of a research project on local socio-environmental problems conducted with adult literacy students in situations of exclusion}

Carine VILLEMAGNE

Sherbrooke University, Québec, Canada

The notion of sharing the planet does not always mean sharing it with others. People living in the downtown areas of densely populated cities can lead very solitary lives. Certain populations are susceptible to living in isolation and in poor-quality conditions without any opportunity for expression or supportive relationships. These disadvantaged populations are those most often confronted with social and ecological disorder.

The research project, conducted in collaboration with two community organizations and a university, consists of designing and using the educational program "Literacy and environmental education for adults". This program targets the social and environmental commitment of adult literacy students in their own neighbourhoods. Since the research is in its early stages, the collaborators are working to create a program based on their practical experiences and their theoretical articles, which are reviewed and analyzed. At this stage, several challenges must be overcome in order to develop the program. This article examines some of them, on both practical and theoretical levels, and then presents the general orientations the collaborators want to wish to this program. 


\section{RESUMEN}

\section{Retos y orientaciones principales de un proyecto de investigación arraigado en problemáticas socio-ambientales locales y destinado a adultos poco alfabetizados en situación de exclusión.}

Carine VILLEMAGNE

Universidad de Sherbrooke, Quebec, Canadá

Vivir juntos no siempre significa estar en relación con los otros. Una vida cotidiana muy solitaria puede ser vivida por individuos que residen en ciudades densamente pobladas. Algunas poblaciones son susceptibles de vivir aisladas, sin expresión ni lazos de solidaridad, en medios de vida con muy poca calidad ambiental. Son precisamente esa poblaciones marginales que están más confrontadas con los desordenes sociales y ecológicos. Este proyecto de investigación se realizó en colaboración con dos organismos comunitarios y una universidad y consiste en la concepción y la experimentación del programa educativo «Alfabetización de adultos y educación relativa al entorno ecológico». Este programa tiene como objetivo el compromiso social y ambiental en el medio de vida de adultos poco alfabetizados. Como la investigación se encuentra en sus inicios, los colaboradores trabajan en la concepción del programa basándose en sus experiencias prácticas y en los escritos teóricos inventariados y analizados. En ésta etapa, parece ser que varios retos deben ser resueltos para poder desarrollar el programa. Este artículo examina algunos de ellos, tanto de naturaleza teórica como práctica y presenta las grandes orientaciones que los colaboradores desean imprimir al programa.

\section{Introduction}

Dans les sociétés occidentales d'aujourd'hui, être un adulte analphabète comporte des conséquences qui vont au-delà de ne pas savoir lire, écrire ou simplement compter. Les impacts sont beaucoup plus larges et aliénants, puisqu'ils concernent l'ensemble des dimensions de sa vie. En effet, non seulement l'alphabétisme a des répercussions sur le niveau socioéconomique de l'adulte, mais il influence aussi ses conditions de vie ainsi que sa capacité à exercer ses rôles sociaux. La situation d'analphabétisme augmente les risques d'exclusion (RGPAQ, 2006) : elle empêche potentiellement l'adulte de développer des formes d'engagement sur des questions qui le touchent dans son quotidien. En somme, si l'analphabétisme marginalise certains adultes ou les rend à risque d'être marginalisés dans la société, l'alphabétisation des adultes peut contribuer à ce qu'ils soient capables de s'engager dans leur milieu sur des questions à la fois sociales, communautaires, politiques, 
économiques, culturelles et environnementales. À ce titre, l'alphabétisation populaire au sein des organisations communautaires joue un rôle crucial dans la lutte contre l'analphabétisme, en proposant notamment des formes conscientisantes et émancipatrices de l'alphabétisation.

En ce qui a trait aux questions environnementales, les problèmes sont grandissants et sont autant locaux que mondiaux. Toutes les populations sont touchées par ces problèmes, mais certains groupes sociaux et certains milieux de vie le sont plus que d'autres. Il existe en effet une relation étroite entre les caractéristiques socioéconomiques des adultes et la qualité de leur environnement de vie, qualité qui peut affecter la santé humaine. Les communautés défavorisées sont celles dont les milieux de vie sont le plus souvent fortement dégradés (Malone, 1999). De la même manière, les populations les plus vulnérables sont aussi souvent les plus faiblement scolarisées. Les injustices sociales, économiques et environnementales semblent donc aller de pair (Hillman, 2002).

C'est dans ce contexte socioécologique - ici rapidement esquissé - que s'inscrit le projet de recherche "Alphabétisation et éducation relative à l'environnement (ERE) des adultes", intégrant ensemble les champs théorique et pratique de l'alphabétisation des adultes et l'éducation relative à l'environnement. Ce projet, qui consiste en la conception et en l'expérimentation d'un programme éducatif, poursuit l'objectif général de développer l'engagement social et environnemental de personnes adultes peu alphabétisées en situation d'exclusion sociale. Reposant sur des problématiques socio-environnementales locales et ancré dans le milieu de vie des adultes participants, ce programme "Alphabétisation et ERE des adultes » est axé sur le «faire-vivre» ensemble une expérience concrète de participation des adultes à la vie collective locale. Le projet, qui a également pour objectif de contribuer à une transformation libératrice des personnes participantes, vise à influencer leur rapport au milieu de vie. Il veut aider les adultes à prendre leur place dans leur communauté. La démarche de recherche adoptée est celle d'une «recherche de développement d'objet éducatif» (Loiselle, 2001) où les données recueillies tout au long de la recherche renseignent autant sur le processus que sur les résultats de celle-ci. Ainsi, comme le souligne Loiselle (2001), cette recherche n'est pas sous «le signe de l'empirisme et de l'artisanat». Elle vise à ancrer la pratique éducative dans les résultats des recherches scientifiques menées en éducation, tout autant qu'à produire de nouvelles connaissances au sujet de ce que Loiselle (2001) désigne comme les caractéristiques essentielles, novatrices et transférables de l'objet éducatif, mais aussi les particularités de la démarche de développement de cet objet. Dans notre recherche, l'objet est un programme éducatif croisant l'alphabétisation et l'ERE des adultes. La collecte de données de recherche repose sur plusieurs stratégies: tests de niveau d'alphabétisation et entrevues semi-dirigées avec les adultes participants; groupes de discussion avec les organismes communautaires et avec les adultes participants; analyse documentaire et observation participante durant l'expérimentation du programme.

Cette recherche est planifiée sur une période de trois ans en plusieurs phases interreliées (Loiselle, 2001; Van der Maren, 2003). La première phase, qui vient de se 
terminer, a consisté en l'analyse de la situation de départ. Il s'agissait de clarifier, et de négocier entre les collaborateurs de la recherche, les objectifs et les besoins auxquels répond la recherche ainsi que les intentions spécifiquement liées au programme «Alphabétisation et ERE des adultes ». L'établissement d'un climat propice au dialogue a aussi été au cœur des premières rencontres entre les collaborateurs. Cette première phase inclut également la recension et l'analyse critique des différents programmes éducatifs existants en vue d'inspirer le programme à concevoir. La deuxième phase de la recherche est en cours. Elle correspond à la conception du programme éducatif "Alphabétisation des adultes et ERE en milieu communautaire». Plusieurs scénarios sont ainsi en construction. La troisième phase consistera en l'expérimentation de ce programme. Et la dernière phase reposera sur la réalisation des activités de transfert et de diffusion du programme «Alphabétisation et ERE des adultes".

À cette étape d'avancement de la recherche, plusieurs défis sont à relever pour mettre en œuvre le programme éducatif. Nous en examinerons quelques-uns, certains de nature théorique, d'autres de nature pratique. Ces défis sont le reflet de la complexité du projet, en raison du croisement de plusieurs champs de recherche, du caractère pragmatique de cette recherche de développement et de sa dimension collaborative, associant deux organismes communautaires et une université. Nous présenterons ensuite les grandes orientations que les deux organismes communautaires collaborateurs souhaiteraient donner à ce programme éducatif (Villemagne, Daniel, Guijarro, Lemieux et Nault, 2009). Ces dernières sont importantes, puisqu'elles ont servi à l'examen critique des différents programmes éducatifs recensés durant la phase 1 et qu'elles inspirent les scénarios du programme en construction.

\section{Quelques défis associés au projet de recherche "Alphabétisation et ERE des adultes »}

Les défis associés au projet de recherche "Alphabétisation et ERE des adultes " sont multiples. Nous en explorons quelques-uns dans cet article. Ces défis ont été mis évidence par l'analyse de plusieurs types de données recueillies: 1) les écrits scientifiques recensés lors d'une revue de littérature; 2 ) les comptes rendus de réunion avec les collaborateurs de la recherche; 3 ) et le verbatim du premier groupe de discussion réalisé avec les collaborateurs.

\section{Défis d'ordre théorique}

D'un point de vue théorique, l'éducation relative à l'environnement des adultes est peu développée : l'ERE est demeurée axée sur les jeunes générations plus que sur les adultes. Pourtant, ces derniers sont les décideurs d'aujourd'hui en matière d'environnement, influençant le présent et le futur des êtres vivants et de leurs écosystèmes. Et lorsque ces propositions d'ERE des adultes existent, elles sont très éclatées, allant de l'énoncé de simples principes comme ceux de Finger (1989) à une contribution plus développée et fondée comme celle de Clover et de ses collaborateurs 
Défis et principales orientations d'un projet de recherche ancré dans des problématiques socio-environnementales locales et destiné à des adultes peu alphabétisés en situation d'exclusion

Puisque les adultes sont reconnus pour avoir une conscience environnementale moins développée que les jeunes, la création d'un programme éducatif « Alphabétisation et ERE des adultes » ancré dans les préoccupations socio-environnementales des acteurs locaux et des adultes eux-mêmes constitue un intéressant levier de changement dans leur milieu de vie. $\left(2000^{1}\right)$ (Villemagne, 2005, 2008a). Puisque les adultes sont reconnus pour avoir une conscience environnementale moins développée que les jeunes, la création d'un programme éducatif «Alphabétisation et ERE des adultes» ancré dans les préoccupations socio-environnementales des acteurs locaux et des adultes eux-mêmes constitue un intéressant levier de changement dans leur milieu de vie. Par ailleurs, les points d'arrimage de l'ERE des adultes avec le champ de l'éducation des adultes et spécifiquement de l'alphabétisation des adultes ont été peu explorés jusqu'à ce jour (Villemagne, 2008b). Les propositions théoriques pouvant servir de fondements à l'élaboration du programme éducatif "Alphabétisation et ERE des adultes" demeurent ainsi à construire. Une telle entreprise semble possible car certains courants de l'ERE (Sauvé, 2003) comme de l'alphabétisation présentent des fondements théoriques compatibles. C'est le cas par exemple du courant de la critique sociale en ERE et du courant de l'alphabétisation conscientisante et émancipatrice.

\section{Défis d'ordre pratique}

Les deux organismes communautaires locaux sollicités pour le projet de recherche sont issus à la fois du domaine « social » et du domaine « environnemental ». Ils n'ont pas d'expériences communes de collaborations antérieures. Si Kempf (2007) milite en faveur d'une mise en relation systématique de l'écologie et du social dans tous les choix de société, il n'en demeure pas moins que faire travailler ensemble divers types d'acteurs dont les missions respectives sont historiquement dissociées, peut constituer une entreprise délicate. Les groupes communautaires collaborant à ce projet de recherche devront donc apprendre à se comprendre en clarifiant leurs présupposés, leurs représentations, leurs systèmes de valeurs respectifs et apprendre à développer un langage commun; ils devront également négocier des types de collaboration qui respectent les contraintes qui leur sont propres.

Mentionnons aussi un autre défi d'ordre pratique. Les quelques pratiques éducatives mises en œuvre jusqu'ici dans le champ de l'ERE des adultes sont jugées pauvres et instrumentales (Clover, 1995) : il ne suffit pas d'informer les adultes sur les questions d'environnement, de développer leur savoir pour qu'ils veuillent nécessairement prendre part à ces questions et s'engager dans des changements réfléchis et concrets. Par ailleurs, ces pratiques éducatives seraient non participatives, en ce sens que les adultes ne sont ni actifs ni engagés dans le processus éducatif. Ces pratiques non participatives pourraient ne pas contribuer au développement d'une éducation relative à l'environnement des adultes qui soit suffisamment signifiante pour pousser les adultes à s'engager individuellement et collectivement en matière d'environnement. Une première recension en cours de programmes éducatifs existants et pouvant inspirer le programme à concevoir confirme le nombre limité de sources pertinentes.

Par ailleurs, lors d'une étude exploratoire réalisée dans plusieurs régions administratives québécoises auprès de 21 organismes d'alphabétisation populaire, nous

1. Dans Villemagne (2008a), nous avons réalisé une revue détaillée de plusieurs propositions de l'ERE des adultes. C'est la raison pour laquelle nous n'y reviendrons pas dans cet article. 
Les groupes d'alphabétisation populaire se sentent peu outillés pour intégrer l'éducation relative à l'environnement (ERE) à leurs pratiques éducatives. n'avons pu repérer aucune pratique d'alphabétisation qui s'intéresse aux questions environnementales (Villemagne et Allaire, 2008). Cette étude montre néanmoins que $85 \%$ des organismes expriment le souhait de développer de telles pratiques, en raison de l'urgence d'agir sur certaines problématiques environnementales qui affectent les adultes, de leur sentiment de responsabilité collective à l'égard de l'environnement, mais aussi en raison des demandes formulées par les adultes eux-mêmes. Les modalités d'intégration des questions environnementales à l'éducation des adultes pourraient être diversifiées : par exemple, utiliser l'environnement comme simple support de lecture ou élaborer des projets de résolution de problèmes socioenvironnementaux concrets. Néanmoins, les groupes d'alphabétisation populaire se sentent peu outillés pour intégrer l'éducation relative à l'environnement (ERE) à leurs pratiques éducatives. Parmi les quelques raisons invoquées, figurent le manque de formation des formateurs et surtout l'absence de matériel éducatif adapté à l'organisation d'ateliers concrets pour des personnes peu ou pas alphabétisées (Villemagne et Allaire, 2008). Le projet "Alphabétisation et ERE des adultes» tente ainsi de proposer une réponse aux besoins énoncés par des acteurs de terrain.

\section{Les principales orientations suggérées par les collaborateurs de la recherche}

Les principales orientations résultent de l'analyse qualitative d'un compte rendu de réunion dressé avec les collaborateurs de la recherche et du verbatim d'un premier groupe de discussion réalisé avec ces mêmes collaborateurs. Pour désigner chacun des deux organismes qui collaborent au projet de recherche, nous utiliserons respectivement les expressions organisme "social» pour l'organisme communautaire à vocation sociale et organisme « environnemental » pour l'organisme communautaire à vocation environnementale.

\section{Partir des expertises éducatives et environnementales propres aux organismes... pour les dépasser Expertises éducatives}

L'organisme social possède une mission d'éducation populaire clairement affichée. Sa visée éducative générale repose sur l'empowerment d'adultes vivant des problèmes d'itinérance et d'exclusion sociale, de même que sur le développement et l'exercice de leur citoyenneté. Les adultes fréquentant l'organisme social sont ainsi invités à participer à des cafés-rencontres, à des comités de travail et à des processus décisionnels qui concernent la défense et la revendication de leurs droits. Ces activités proposées aux adultes sont en général de nature participative et sont orientées vers l'action.

Pour l'organisme environnemental, les activités éducatives portent surtout sur l'«information » et la « sensibilisation » de leurs membres. Elles sont réalisées dans le cadre de la mission environnementale de l'organisme. À cet effet, l'organisme environnemental conçoit son intervention en trois temps : faire prendre conscience aux 
membres des différents problèmes environnementaux existants, leur faire identifier des actions à entreprendre et les mettre en application (AMTE, 2009). Le volet d'éducation relative à l'environnement demeure encore embryonnaire, mais l'organisme environnemental désire le développer selon une double perspective biorégionale et communautaire.

Les expertises éducatives de chacun des groupes sont fort différentes, que ce soit en matière de public cible ou même d'actions éducatives proprement dites. L'établissement d'un dialogue entre les parties est donc important : un dialogue pour se comprendre, un dialogue pour partager son expertise respective. Ainsi, même si les expertises éducatives diffèrent, il semble évident que les deux groupes communautaires tendent à développer des activités éducatives qui visent l'engagement dans l'action des participants.

\section{Expertises environnementales}

L'organisme social présente un volet alimentaire très marqué dans sa mission, puisqu'il cuisine et offre des repas à ses membres en situation de pauvreté. Cet organisme souhaite ainsi agir à titre préventif au regard des problèmes de santé liés à l'alimentation des personnes adultes itinérantes. Une telle mission a amené les responsables à réfléchir sur l'empreinte écologique de l'organisme et à introduire le recyclage et le compostage dans les activités de production de repas. Un jardin collectif est également réalisé chaque année avec l'aide des membres. La volonté actuelle est de développer plus activement des actions d'ERE directement auprès des membres, afin que ces derniers intègrent une préoccupation environnementale dans leur quotidien.

Quant à l'organisme environnemental, sa mission est d'offrir aux citoyens la possibilité de se regrouper pour traiter des questions environnementales. Parmi ces

Les questions d'alimentation, d'agriculture ainsi que la gestion des déchets organiques et non organiques sont des dimensions privilégiées par les deux organismes communautaires collaborateurs du projet de recherche. questions, on retrouve l'agriculture urbaine, le transport, l'énergie, les déchets, la mise en marché et l'accès des citoyens à des produits sains et locaux, etc. Son projet le plus abouti est celui qui favorise l'accès des citoyens à une alimentation saine et locale, à travers l'établissement d'un marché de solidarité régionale. Avec ce projet, l'organisme environnemental souhaite contribuer au développement d'une agriculture respectueuse de l'environnement ainsi qu'au développement d'une économie locale et solidaire.

Les questions d'alimentation, d'agriculture ainsi que la gestion des déchets organiques et non organiques sont des dimensions privilégiées par les deux organismes communautaires collaborateurs du projet de recherche. Néanmoins, elles ne sont pas traitées de la même manière. Pour l'organisme social, il s'agit de contribuer à satisfaire des besoins de base des adultes. Manger à sa faim fait en effet partie des besoins physiologiques primaires de l'humain, dont la satisfaction est nécessaire à sa survie et au maintien d'un état de santé physique. Pour l'organisme environnemental, ses membres n'ont en général pas à relever le défi de leur survie (manger). Il s'agit pour ce groupe de participer à transformer les pratiques alimentaires de ses membres en tenant compte des impacts environnementaux exercés par la production, la transformation et la distribution des denrées alimentaires. 


\section{Prendre en compte les caractéristiques et les besoins des adultes ciblés}

Les deux organismes communautaires collaborateurs s'accordent sur la nécessité de prendre en compte les caractéristiques et les besoins des adultes ciblés par le projet de recherche, adultes qui seront a priori ceux fréquentant l'organisme social. En ce qui concerne les besoins, bien que plusieurs soient d'ores et déjà définis par les groupes collaborateurs de la recherche, il conviendra également de consulter les adultes participant au programme éducatif lorsque ceux-ci auront été recrutés.

\section{Caractéristiques des adultes ciblés}

Rappelons que le projet de recherche vise des personnes adultes âgées de 16 à 65 ans qui choisiront volontairement de participer aux activités du programme "Alphabétisation et ERE des adultes». Ces adultes auront été repérés au sein de l'organisme social collaborateur de la recherche, en raison de leur faible niveau d'alphabétisme (qui sera ensuite précisé pour chacun des adultes lors de rencontres individuelles). En effet, les adultes ciblés par notre recherche seront des personnes bénéficiant des activités et des services offerts par ce groupe. Le niveau d'alphabétisme de ces personnes sera variable d'un adulte à un autre. Si les personnes adultes ne sachant ni lire ni écrire sont plutôt rares au Québec, il est néanmoins constaté que de faibles habitudes de lecture et d'écriture, une mauvaise compréhension en lecture et une peur d'écrire caractérisent les adultes fréquentant l'organisme social. Il faudra donc tenir compte dans le programme éducatif à concevoir que les participants ne sont pas des personnes totalement analphabètes mais qu'elles vivent des problématiques multidimensionnelles.

Ainsi, les adultes qui participeront au projet de recherche connaissent des problèmes d'itinérance et d'exclusion sociale. Considérées comme des problèmes sociaux très complexes, l'itinérance et l'exclusion sociale présentent différentes facettes qui, au quotidien, auront un effet sur la participation des adultes au programme éducatif conçu dans la cadre de la recherche. Nous avons ainsi retenu deux autres caractéristiques à prendre en compte dans la conception de ce programme : le milieu de vie des adultes itinérants correspond à l'environnement immédiat de l'organisme social qu'ils fréquentent, et non pas à un quartier spécifique, dans le cas où ils posséderaient un logement fixe. Ces adultes éprouvent en général un fort sentiment d'appartenance à leur groupe social, appartenance qui sera donc à valoriser. Par ailleurs, il existe un important «roulement» chez les adultes qui fréquentent l'organisme social, ce qui a un effet sur les projets planifiés en plusieurs rencontres. Il n'est pas assuré que les participants seront présents à toutes les rencontres. Or, le programme «Alphabétisation et ERE des adultes» se déroulera sur une période de 3 à 4 mois. Maintenir la participation des adultes dans le cadre du projet de recherche sera donc un défi à relever. Il faudra s'assurer d'avoir un noyau central de 3 ou 4 personnes présentes durant tout le projet. D'autres personnes dites «satellites » pourraient n'être présentes qu'à certaines étapes de celui-ci. 
Défis et principales orientations d'un projet de recherche ancré dans des problématiques socio-environnementales locales et destiné à des adultes peu alphabétisés en situation d'exclusion

\section{Besoins des adultes ciblés}

Les adultes ciblés par le projet de recherche ont en général un double besoin de reconnaissance personnelle et sociale qu'il serait fort pertinent d'intégrer dans le programme éducatif à concevoir.

Les collaborateurs de la recherche proposent ainsi d'explorer les possibilités de valorisation de la participation des adultes au programme éducatif sous forme de reconnaissance des apprentissages réalisés (crédits de formation, par exemple); les collaborateurs suggèrent aussi de médiatiser au sein de la population locale les formes d'engagement socio-environnemental des adultes qui émergeront du projet. Une telle médiatisation vise à défaire les préjugés liés à l'itinérance ou à la pauvreté : par exemple, les personnes pauvres ne souhaiteraient pas participer à la résolution de problèmes environnementaux locaux (parce qu'elles sont trop accaparées par d'autres types de problèmes? parce qu'elles sont peu éduquées? pour d'autres raisons?).

\section{Caractéristiques du programme éducatif à concevoir Un programme orienté vers l'action}

Pour motiver les adultes à participer aux différentes étapes du programme éducatif, les deux organismes communautaires collaborateurs au projet de recherche considèrent que la tenue d'ateliers théoriques n'est pas pertinente; il est important d'orienter les différentes activités éducatives vers l'action, en intégrant dans le projet les adultes participants. La possibilité de lancer le projet par une activité de déclenchement comme la prise de photographies dans leurs quartiers a été formulée. Les photographies devraient illustrer soit un environnement de qualité, soit un environ-

Il semble pertinent pour les collaborateurs de créer des liens entre les adultes participants et la population locale. Il s'agit de participer ainsi à la lutte contre l'exclusion sociale des adultes et de contribuer

à leur reconnaissance par la population locale, en contribuant à briser les préjugés dont les adultes itinérants sont l'objet. nement dégradé. Ces photos pourraient ensuite servir de point de départ à une discussion de groupe sur les photographies prises individuellement. L'idée de monter une exposition des photographies dans un lieu public ou semi-public du quartier où les adultes présenteraient à la population locale leur vision de l'environnement et les projets possibles qui pourraient en découler a aussi été émise.

\section{Un programme ancré dans la communauté locale}

Il semble pertinent pour les collaborateurs de créer des liens entre les adultes participants et la population locale. Il s'agit de participer ainsi à la lutte contre l'exclusion sociale des adultes et de contribuer à leur reconnaissance par la population locale, en contribuant à briser les préjugés dont les adultes itinérants sont l'objet. Le sentiment d'utilité sociale peut aussi être amélioré par le la mise en œuvre d'une action socio-environnementale par les adultes, action ayant des retombées dans le milieu de vie.

\section{Un programme facile d'appropriation pour les collaborateurs}

La collaboration des deux organismes communautaires au projet de recherche devrait avoir des retombées positives pour ces derniers. Les organismes communautaires souhaiteraient pouvoir s'approprier certaines activités du programme «Alphabétisation et ERE des adultes» (en les adaptant au besoin) en vue de les intégrer 
à leur programmation annuelle. Les organismes communautaires souhaiteraient aussi explorer d'autres approches ou stratégies éducatives que celles qu'ils utilisent déjà, afin d'enrichir ou de transformer leur savoir-faire.

\section{Conclusion}

Le projet de recherche «Alphabétisation et ERE des adultes » présente ainsi de multiples défis mais ces derniers sont, croyons-nous, surmontables. De nouvelles façons de travailler sont à imaginer et il reste à élaborer une culture commune de recherche et de pratique au fil des rencontres entre les collaborateurs. Cette recherche «terrain » comporte en effet une dimension collaborative, en ce sens que les acteurs communautaires qui collaborent à la recherche ont un rôle majeur à jouer. Les savoirs qui émergeront de la recherche seront "co-construits » par l'équipe universitaire et les organismes communautaires reconnus comme des acteurs sociaux compétents (Desgagné et collab., 2001; 1998).

Les types de participants ciblés par le programme éducatif engendrent aussi des défis d'une autre nature. En raison de la réalité communautaire locale dans laquelle s'insère le projet de recherche, nous rejoindrons des personnes peu alphabétisées, certes, mais ces personnes sont également aux prises avec les problématiques supplémentaires de l'itinérance, phénomène social multifactoriel et dynamique à l'endroit duquel de nombreux préjugés et des croyances sont exprimés par les citoyens (MSSS, 2008).

Bien des interrogations demeurent sur la forme concrète finale du programme éducatif envisagé. S'il apparaît que l'action doit être placée au cœur de celui-ci, il n'est pas encore possible de préciser cette action. Certaines approches éducatives seront a priori privilégiées, en particulier les approches expérientielles conscientisantes et communautaires, visant à développer le réseau de relations adultes-communautémilieu de vie. Il s'agira ainsi de développer une conscience critique chez l'adulte, à travers le dialogue avec ses pairs, de certaines réalités socio-environnementales qui le concernent. Il s'agira aussi de déconstruire les présupposés qui fondent ces réalités (ce que Finger et Asun [2001] nomment, pour leur part, la clarification conceptuelle). Il s'agira ensuite d'imaginer d'autres possibles par le développement de projets concrets ancrés dans la communauté locale, projets où l'empowerment de l'adulte sera au cœur du processus éducatif (Le Bossé et Dufort, 2001). 


\section{Références bibliographiques}

AMIS DE LA TERRE DE L'ESTRIE (2009). La mission de l'organisme. Document interne. Sherbrooke: AMTE.

CLOVER, D.E. (1995). Theoretical Foundations and Practice of Critical Environmental Adult Education in Canada. Convergence, 28(4), p. 44-54.

CLOVER, D., FOLLEN, S. et HALL, B. (2000). The Nature of Transformation. Environmental Adult Education ( $2^{\mathrm{e}}$ éd.). Toronto : Ontario Institute for Studies in Education.

DESGAGNÉ, S. (2001). La recherche collaborative : nouvelle dynamique de recherche en éducation, dans M. Anadón (dir.), Nouvelles dynamiques de recherche en éducation Québec: Les Presses de l'Université Laval, p. 51-76.

DESGAGNÉ, S. (1998). Réflexions sur le concept de recherche collaborative, dans N. Bednarz (dir.), Recherche collaborative et partenariat: quelques notes et réflexions (p. 31-46). Montréal : CIRADE.

FINGER, M. (1989). Environmental Adult Education from the Perspective of the Adult Learner. Convergence, 22(4), p.25-32.

FINGER, M. et ASUN, J.M. (2001). Adult Education at the Crossroads. Learning Our Way Out. Londres : Zed Books.

HILLMAN, M. (2002). Environmental Justice: A Crucial Link between Environmentalism and Community Development? Community Development Journal, 37(4), p.349-360.

KEMPF, H. (2007). Comment les riches détruisent la planète. Paris : Seuil

LE BOSSÉ, Y. et DUFORT, F. (2001). Le pouvoir d'agir (empowerment) des personnes et des communautés, dans F. Dufort et J. Guay (dir.), Agir au cœur des communautés. La psychologie communautaire et le changement social. Québec: Les Presses de l'Université Laval.

LOISELLE, J. (2001). La recherche-développement en éducation: sa nature et ses caractéristiques, dans M. Anadon et M. L'Hostie, Nouvelles dynamiques de recherche en éducation. Québec: Les Presses de l'Université Laval, p. 77-97.

MALONE, K. (1999). Reclaiming Silented Voices through Practices of Education and Environmental Popular Knowledge Production. Canadian Journal of Environmental Education, 4, p. 232-242.

MINISTERE DE LA SANTE ET DES SERVICES SOCIAUX (2008). L'itinérance au Québec: cadre de référence. Québec: MSSS, Gouvernement du Québec.

SAUVÉ, L. (2003). Courants et modèles d'intervention en éducation relative à l'environnement. Module 5. Programme d'études supérieures - Formation en éducation relative à l'environnement - Francophonie internationale. Montréal : Les Publications ERE-UQAM, Université du Québec à Montréal Collectif ERE-Francophonie. 
VAN DER MAREN, J.-M. (2003). La recherche appliquée en pédagogie. Des modèles pour l'enseignement ( $2^{\mathrm{e}}$ éd.). Bruxelles : De Boeck.

VILLEMAGNE, C. (2005). L'éducation relative à l'environnement en milieu communautaire urbain. Un modèle théorique en émergence enrichi de l'exploration collaborative de pratiques éducatives. Thèse inédite de doctorat. Montréal/Rennes : Université du Québec à Montréal et Université de Haute-Bretagne.

VILLEMAGNE, C. et ALLAIRE, J.-F. (2008). Enquête exploratoire sur les pratiques, intérêts et conceptions en matière d'environnement des groupes d'alphabétisation populaire de trois régions administratives $d u$ Québec, Rapport de recherche - Fonds du CRSH institutionnel. Sherbrooke : Université de Sherbrooke.

VILLEMAGNE, C. (2008a). Regard sur l'éducation relative à l'environnement des adultes. Revue électronique des sciences de l'environnement VertiGO 8(1), 8 p. http://www.vertigo.uqam.ca/vol8nol/art5vol8nol/carine_villemagne.html.

VILLEMAGNE, C. (2008b). L'éducation relative à l'environnement en contexte d'alphabétisation des adultes. Quelles dimensions critiques? Revue internationale francophone en ERE: Regards - Recherches - Réflexions, 7, p.49-64.

VILLEMAGNE, C., DANIEL, J., GUIJARRO, G., LEMIEUX, F. et NAULT, A. (2009). Rencontres préparatoires. Recherche «Alphabétisation et ERE des adultes». Sherbrooke: Université de Sherbrooke.

\section{Remerciements}

Nous remercions André Nault, Géraldine Guijarro et François Lemieux pour leur collaboration au démarrage de cette recherche financée par le Fonds québécois de la recherche sur la société et la culture. Nous remercions aussi les assistantes de recherche Justine Daniel et Caroline Beauregard-Descôteaux dont plusieurs réalisations professionnelles ont contribué à la rédaction de cet article. 